\title{
Use of 18F-FDG-PET/CT for Retroperitoneal/Intra-Abdominal Soft Tissue Sarcomas
}

\author{
Dao-ning Liu $\left(\mathbb{D},{ }^{1}\right.$ Zhong-wu Li $\mathbb{D},{ }^{2}$ Hai-yue Wang $\mathbb{D},{ }^{2}$ Min Zhao $\mathbb{D}^{2},{ }^{2}$ Wei Zhao $\mathbb{D},{ }^{3}$ \\ and Chun-yi Hao ${ }^{1}{ }^{1}$ \\ ${ }^{1}$ Sarcoma Center, Peking University Cancer Hospital \& Institute, Beijing, China \\ ${ }^{2}$ Department of Pathology, Peking University Cancer Hospital \& Institute, Beijing, China \\ ${ }^{3}$ Department of Nuclear Medicine, Peking University Cancer Hospital \& Institute, Beijing, China
}

Correspondence should be addressed to Chun-yi Hao; haochunyi@bjmu.edu.cn

Received 22 March 2018; Accepted 24 May 2018; Published 2 July 2018

Academic Editor: Elena Bonanno

Copyright ( 92018 Dao-ning Liu et al. This is an open access article distributed under the Creative Commons Attribution License, which permits unrestricted use, distribution, and reproduction in any medium, provided the original work is properly cited.

Rationale. To assess the diagnostic value of 18F-FDG-PET/CT for different retroperitoneal soft tissue sarcomas (STS) and other similar tumors. To analyze the predictive value of 18F-FDG-PET/CT for histological grade and main prognostic factors. Methods. 195 patients with 44 different diseases have been included. Relationship between SUVmax, Clinical, pathological, and prognostic information has been analyzed. Results. Malignant tumors do not show higher SUVmax than benign ones $(P=0.443)$. We divided all 44 different diseases into two groups; SUVmax of group 1 is significantly higher than group $2(P \leq 0.001)$. The ROC curve suggests 4.35 is the cutoff value to distinguish groups 1 and 2 (sensitivity $=0.789$; specificity $=0.736$ ). SUVmax correlates with Ki67 index, mitotic count, vascular resection, histological grade, and recurrent STS without considering pathological diagnosis $(P=0.001, P=0.012, P=0.002, P \leq 0.001$, and $P=0.037$, resp.). Conclusion. 18F-FDG-PET/CT cannot simply distinguish malignant and benign tumors in retroperitoneal/intra-abdominal cavity; however, the SUVmax of malignant tumors, inflammatory pseudotumor, and PPGL group is higher than the SUVmax of benign tumors, lymph node metastasis, hematoma, and low malignant STS group. Guidance of "SUVmax location" may be helpful for biopsy and pathology dissection.

\section{Introduction}

Retroperitoneal and intra-abdominal sarcomas contain various soft tissue tumors and a wide prognostic range. Precise diagnosis of these sarcomas always plays a key role in treatment selection, especially in the application of compartment resection [1]. As STS are generally cured by adequate surgical resection, inaccurate diagnosis may cause unnecessary resection of innocent organs and extra risks. Judgment of malignancy is not accurate even using biopsy [2]. Moreover, some researchers resort to other preoperative examinations such as $18 \mathrm{~F}$-Fluoro-2-deoxy-D-glucose (18FFDG) positron emission tomography (PET); however, the effort of 18F-FDG-PET/CT to distinguish extremity lowgrade sarcomas and benign lesions is not fully paid off [3]. Unlike extremity STS, the special anatomical cavity contains many different pathological types that can mimic STS. There is yet no such comprehensive study regarding the use of 18 F-FDG-PET/CT in retroperitoneal and intraabdominal STS, considering the limitation of low incidence of STS [4]. As the establishment of the only sarcoma center in China, the abundant resource provides us an opportunity to afford such an analysis.

The use of 18F-FDG-PET/CT in oncology is based on the FDG accumulation in malignant tumor cells. 18F-FDGPET/CT is initially used for diagnosis, staging, and therapy monitoring. The value for prediction of tumor biology and even prognosis has been found in recent researches $[5,6]$. To evaluate the use of $18 \mathrm{~F}-\mathrm{FDG}-\mathrm{PET} / \mathrm{CT}$ in precise diagnosis and prognosis prediction, we try to correlate maximum standardized uptake value (SUVmax) with different pathologic diagnosis and prognostic factors. In recent researches, common STS prognostic factors are histological grade, tumor size, age, location, vascular resection, number of resected organs, Ki-67 index, and multifocality [7-9]. Among them, Ki-67 is a nuclear protein associated with 

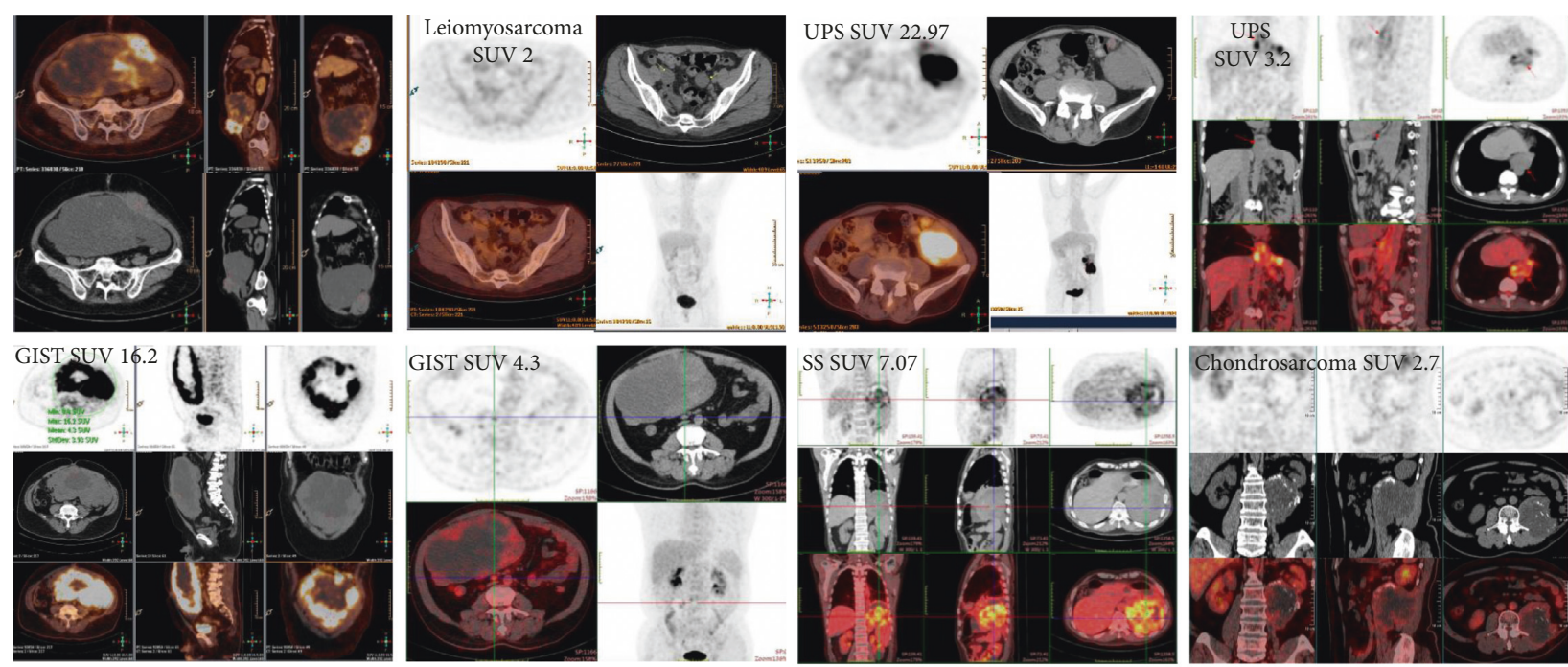

Chondrosårcoma SUV 2.7
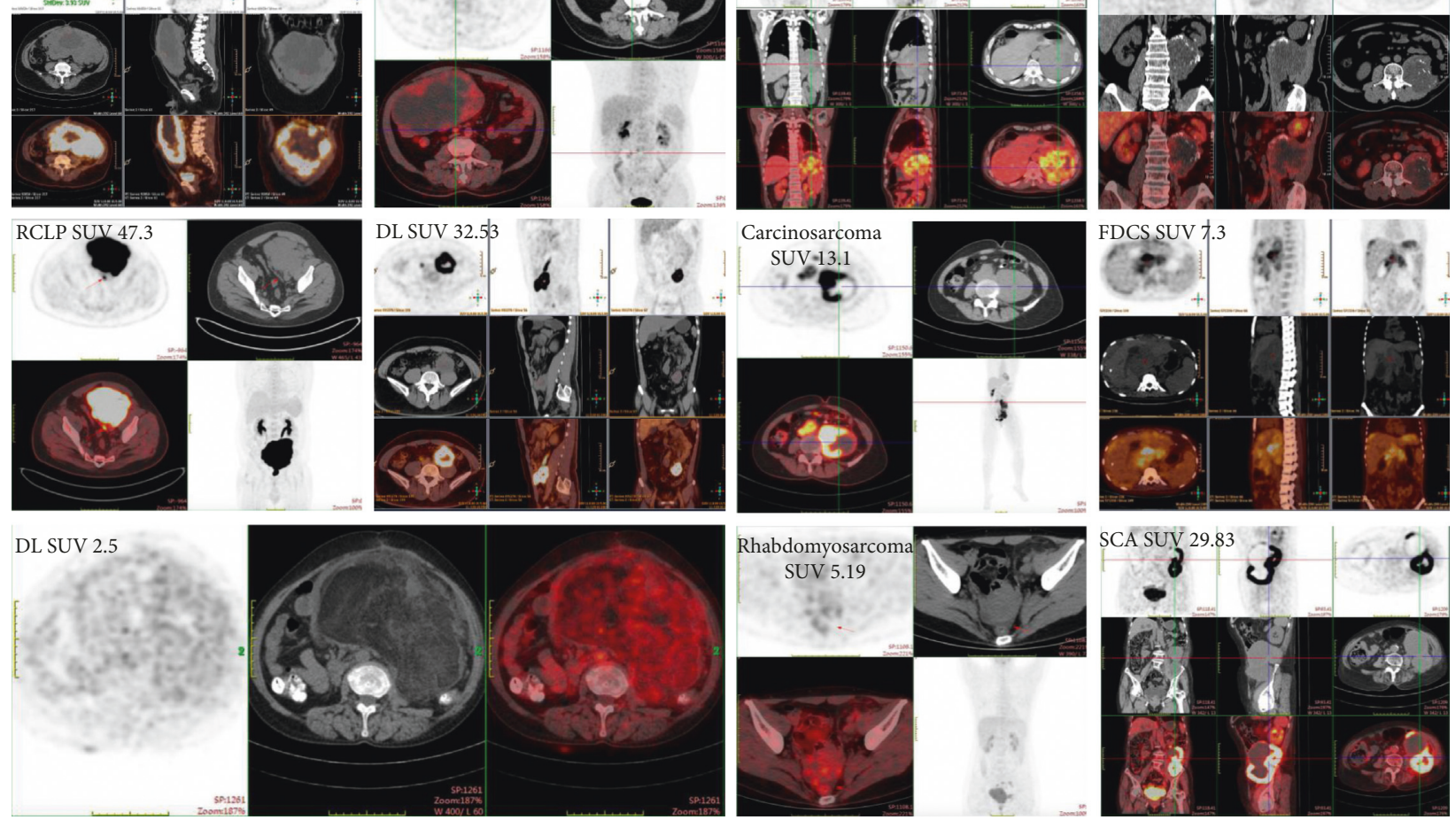

(a)

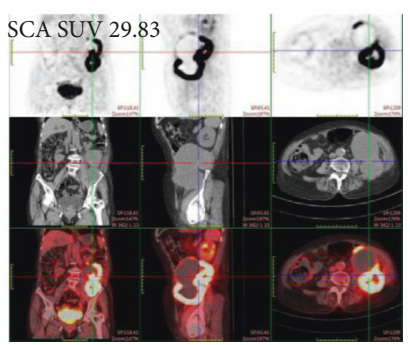

FIgUre 1: Continued. 

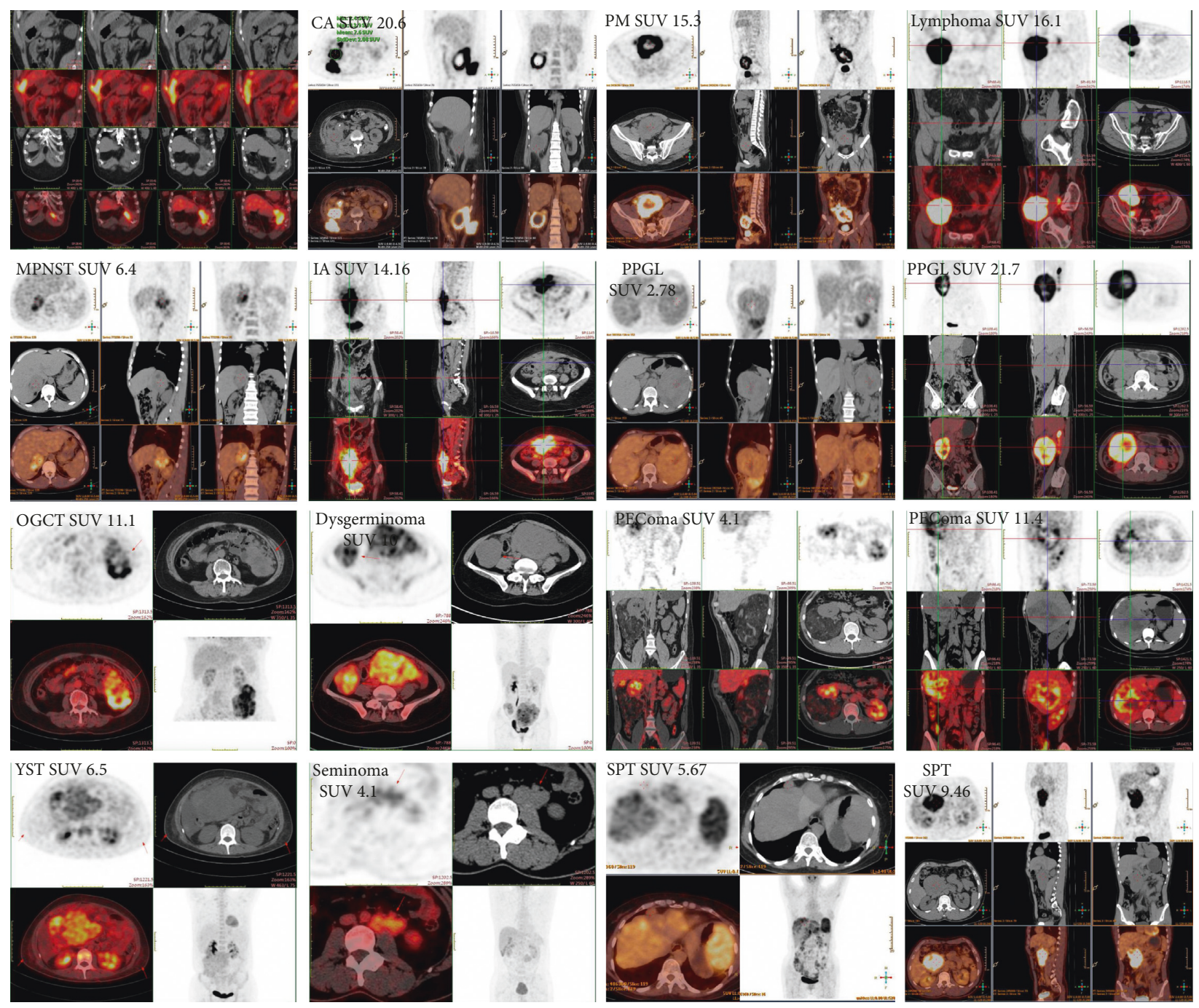

(b)

FIGURE 1: Representative cases in group 1. STS in group 1 are listed in (a). Other tumors in group 1 are listed in (b).

cellular proliferation. Histological grade of the FNCLCC system has been widely used in prognostic prediction for most STS [10]. They will be perfect representative histological data for us to evaluate 18F-FDG-PET/CT.

\section{Materials and Methods}

2.1. Patients. 195 patients with 44 different pathological diagnosis have been enrolled. All patients accepted surgical treatment and 18F-FDG-PET/CT in retroperitoneal and intraabdominal soft tissue sarcoma center, Peking University Cancer Hospital, during a 4-year period (November, 2013, to December, 2017). All patients did not receive any antitumor treatment before the performance of 18F-FDG-PET/CT. Ethical approval and written informed consent have been obtained. Clinical, pathological, and prognostic information have been collected. Histological grade of STS cases without GIST has been reassessed by two experienced pathologists in accordance with the FNCLCC system [10]; the two pathologists were blinded to the findings of clinical and prognostic information.
These patients were divided into two different parts. The first part included 154 retroperitoneal/intra-abdominal STS patients, for whom the relationship among SUVmax, pathological diagnosis, tumor biology, and clinical characters would be analyzed. Then, 32 patients were excluded as per the inclusion and exclusion criteria listed below. The remaining 121 patients would be used to analyze the relationship between SUVmax and prognosis. The second part included 41 patients with benign tumors, psuedotumors, reproductive tumors, and other tumors. They are excellent cases for differentiation.

\subsection{Inclusion and Exclusion Criteria}

(1) Patients whose preoperative diagnosis and postoperative pathology are soft tissue sarcoma will be included; others will not be included for survival analysis.

(2) Patients do not receive any antitumor treatment before 18F-FDG-PET/CT examination. 

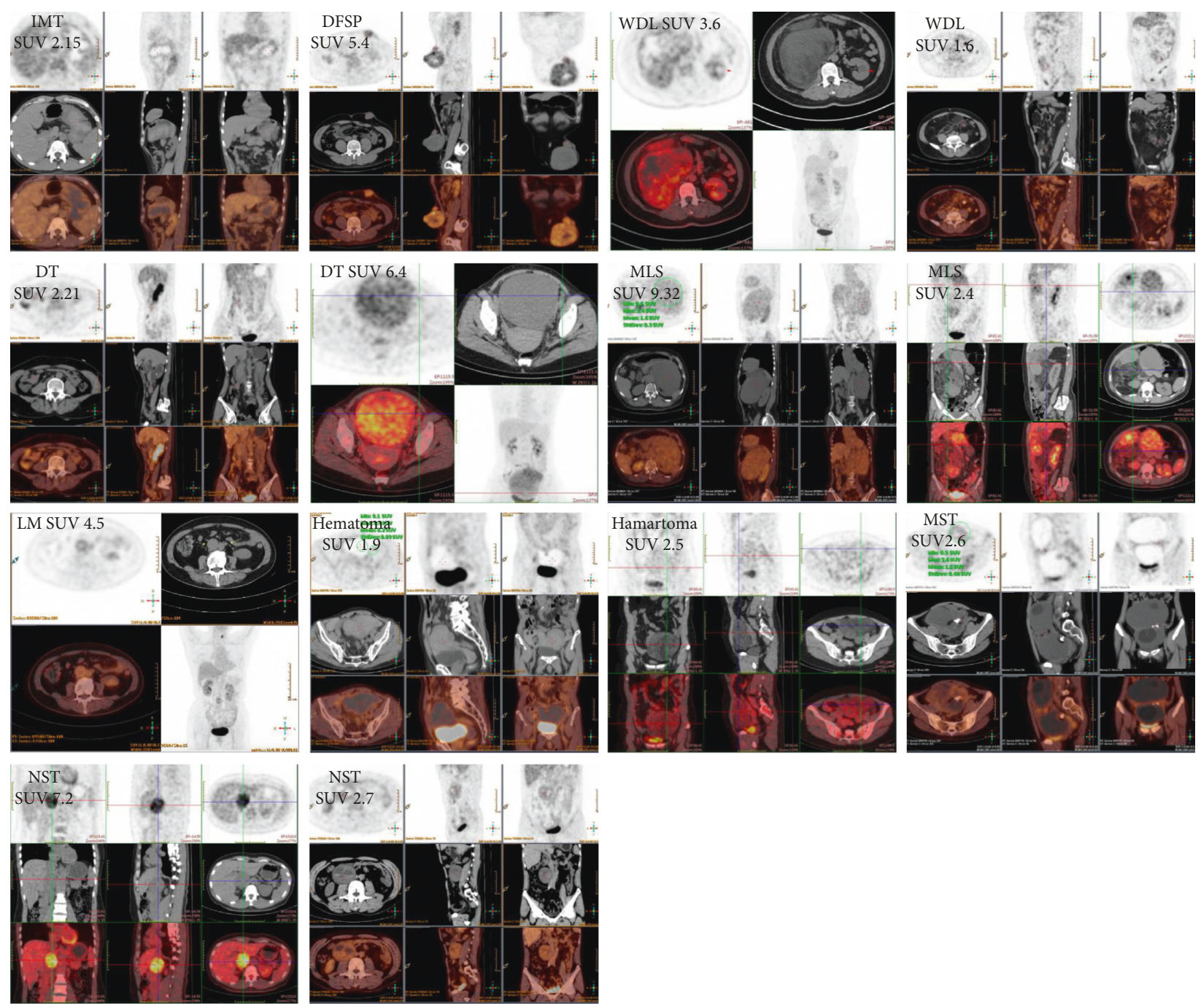

FIgURE 2: Representative cases in group 2.

(3) All patients accept R0/R1 resection, and those who accept R2 resection will be excluded.

(4) Expect for GIST, no distant metastasis is found before/during the operation.

(5) All patients have signed the informed consent and agreed to participate in this study.

(6) Those patients who died of perioperative complications or other noncancer-related causes will be excluded.

2.3. 18F-FDG-PET/CT Acquisition. Patients fasted for at least $6 \mathrm{~h}$ before the 18F-FDG-PET/CT scan. Images were acquired $1 \mathrm{~h}$ after injection of $3.7 \mathrm{MBq} / \mathrm{kg} \quad 18 \mathrm{~F}-\mathrm{FDG}$. Awhole-body scan (brain to midthigh) was performed with the patient in the supine position. CT exposure factors for all scans were $120 \mathrm{kV}$ and $100 \mathrm{mAs}$. 18F-FDG-PET/CT images were reported in consensus by two experienced nuclear medicine physicians, who were blinded to the findings of clinical and prognostic information. At the same time, CT imaging was used to differentiate lesions from physiological uptake. The SUVmax of lesions were calculated. The SUVmax generated from each patient was used in the final analysis.

2.4. Statistics. Data collection and statistical analysis were performed with IBM SPSS Version 20 (SPSS Inc., Chicago, IL, USA). Enumeration data were expressed as mean and standard deviation, ranked data by cross-tabulation and percentages, and survival data by the Kaplan-Meier method. The ROC curve was used to find appropriate cutoff SUVmax for differentiation. For statistical analysis, $T$ test, linear regression, ANOVA, nonparametric test, chi-square test, and log-rank test were employed. All tests were performed twosided at a significance level of $P=0.05$.

\section{Results}

3.1. Diagnosis. For all cases included, SUVmax correlates with Ki-67 index and mitotic count $(P=0.001$, and $P=0.012$, 


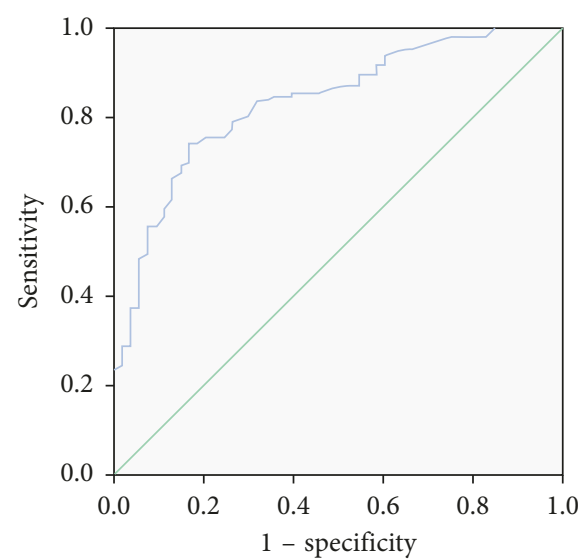

(a)

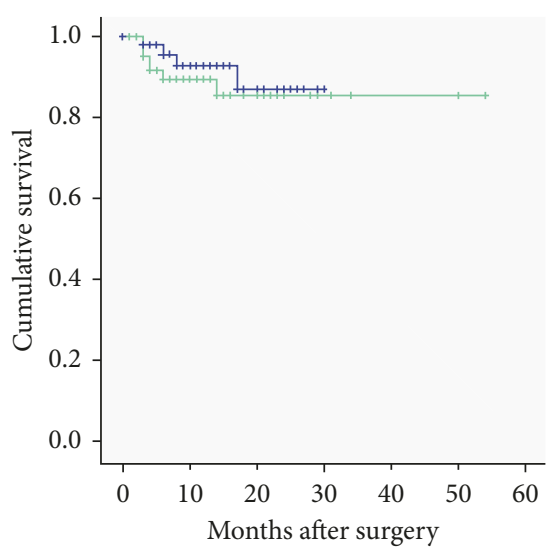

(b)

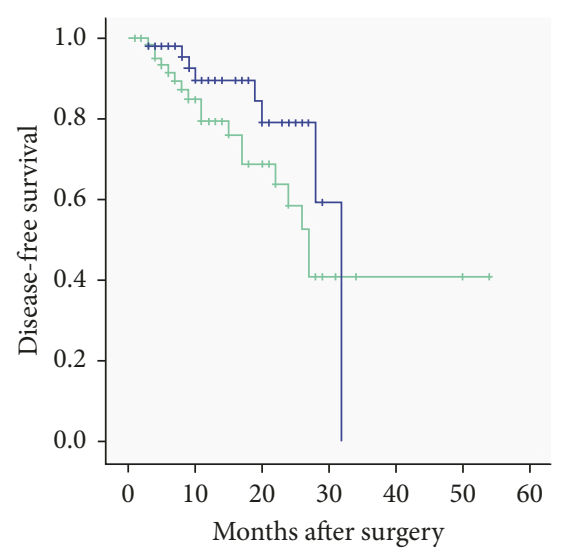

(c)

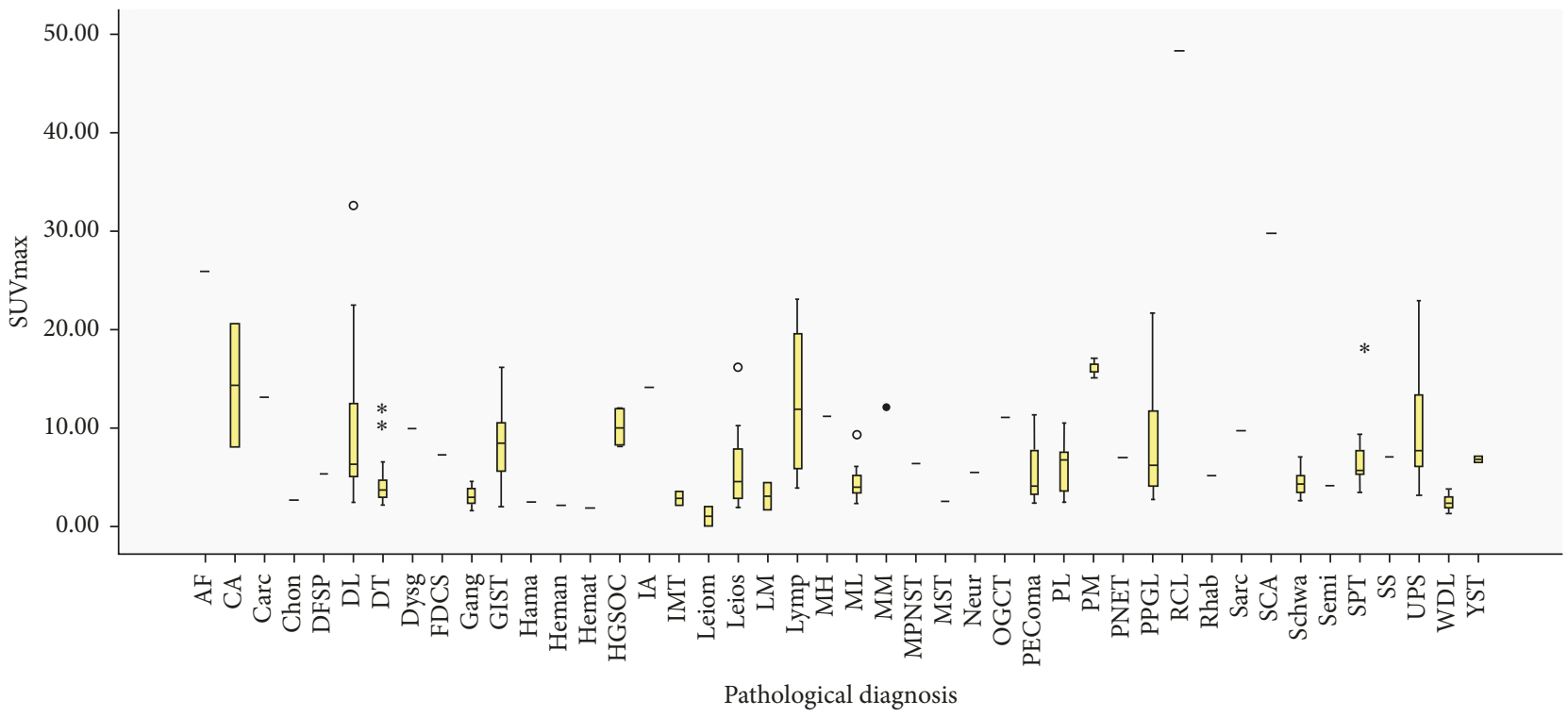

(d)

FIgURE 3: (a) The ROC curve for SUVmax to distinguish group 1 from group 2. (b, c) SUVmax (cutoff at 4.35) does not correlate with OS and DFS using Kaplan-Meier survival curves. (d) The box plot for SUVmax of all cases.

\begin{tabular}{c}
\hline Group 1 Retroperitoneal and intra-abdominal STS \\
Benign tumors \\
Pseudotumor \\
Reproductive tumors \\
Others
\end{tabular}

Group 2 Retroperitoneal and intra-abdominal STS (mainly G1) Benign tumors

Pseudotumor Others

\section{UPS, DL, GIST, SS, PL, leiomyosarcoma, RCL, AF, MH, FDCS, PNET, MPNST, rhabdomyosarcoma, chondrosarcoma PPGL \\ CA, IA, sarcoidosis}

HGSOC, YST, OGCT, dysgerminoma, seminoma

PM, lymphoma,MM, SPT, PEComa, carcinosarcoma, SCA

ML,IMT, WDL, DT, DFSP

Swchannoma, ganglioneuroma, leiomyoma, neurofibroma, MST, hamartoma, hemangioma Hematoma LM

Figure 4: Specific pathological types for groups 1 and 2.

resp.). Malignant tumors do not show higher SUVmax than benign ones $(P=0.443)$. They have been divided into two groups according to the box plot, and literature review, representative images, and pathological types of each group have been shown in Figures 1-4. SUVmax of group 2 is significantly higher than group $1(P \leq 0.001)$. The ROC curve suggests 4.35 is an appropriate cutoff value to distinguish group 1 from group 2 (sensitivity=0.789; 
specificity $=0.736$, Figure 3 ). SUVmax of all diseases are listed in Table 1.

3.2. Treatment. For all STS cases, SUVmax correlates with vascular resection $(P=0.002)$ but has no relationship with combined organs resection (cutoff at 3 organs, $P=0.453$ ). SUVmax does not correlate with pathological invasion of adjacent organs $(P=0.085)$. SUVmax shows no relationship with operative time and blood loss $(P=0.252$ and $P=$ 0.592 , resp.).

3.3. Prognosis. Recurrent STS show higher SUVmax than primitive STS $(P=0.037)$. SUVmax correlates with histological grade $(P \leq 0.001)$, grade 1 is the lowest and grade 3 is the highest. SUVmax for grade 1,2 and 3 are $4.03 \pm 2.28$, $6.31 \pm 4.78$ and $10.09 \pm 12.02$, respectively. SUVmax also significantly correlates with tumor differentiation scores and tumor necrosis scores of the FNCLCC system $(P=$ 0.006 and $P \leq 0.001$, resp.). SUVmax for tumor differentiation scores 1,2 , and 3 are $3.51 \pm 1.99,5.47 \pm 3.84$, and $9.63 \pm$ 7.89 , respectively. SUVmax for tumor necrosis scores 0,1 , and 2 are $5.81 \pm 3.94,9.73 \pm 8.57$, and $11.28 \pm 4.44$. SUVmax shows no significant difference between multifocal and unifocal tumors $(P=0.279)$. SUVmax does not correlate with tumor size $(P=0.279)$. SUVmax shows no relationship with death or postoperative recurrence $(P=0.081$ and $P=0.162$, resp.). Using 4.35 as the cutoff value, SUVmax does not correlate with DFS or OS by the Kaplan-Meier method $(P=0.168$ and $P=0.491$, resp., Figure 3$)$.

\section{Discussion}

Precise preoperative diagnosis of retroperitoneal and intraabdominal sarcomas is always a vital problem, since different pathological diagnosis would lead to completely different treatment and prognosis. In former studies, the intermediate and high-grade malignant lesions have significantly higher FDG-uptake than the low-grade and benign lesions, but 18FFDG-PET/CT offered inadequate discrimination between the latter two groups $[3,11]$. Some researchers also tried to find a cutoff to differentiate malignant from benign tumors; the sensitivity and specificity of 18F-FDG-PET/CT for detecting malignant versus benign lesions were $79 \%$ and $77 \%$ using SUV $\geq 2.0$ and $60 \%$ and $86 \%$ using $\mathrm{SUV} \geq 3.0$, respectively [4].

In our study, we included 44 different diseases for differentiation. Unlike STS elsewhere, we found that 18F-FDG$\mathrm{PET} / \mathrm{CT}$ cannot simply distinguish benign and malignant tumors in retroperitoneal and intra-abdominal cavity. To solve this problem, we divided them into 2 different groups. With this method, we found that sensitivity and specificity for distinguishing 2 different groups are 0.789 and 0.736 using $S U V \max \geq 4.35$. Group 1 stands for malignant tumors, inflammatory pseudotumor, and pheochromocytoma and paraganglioma (PPGL). Group 2 stands for benign tumors, relatively low malignant STS, lymph node metastasis, and hematoma. The theory behind this system is that some STS with relatively low malignancy including desmoid tumor,
TABLE 1: SUVmax for all diseases.

\begin{tabular}{lcc}
\hline Pathological diagnosis & $N$ & SUVmax \\
\hline Retroperitoneal and intra-abdominal STS & & \\
Undifferentiated pleomorphic sarcoma (UPS) & 9 & $10.78 \pm$ \\
Dedifferentiated liposarcoma (DL) & 32 & $8.93 \pm 6.42$ \\
Gastrointestinal stromal tumor (GIST) & 23 & $8.51 \pm 3.73$ \\
Synovial sarcoma (SS) & 2 & $7.09 \pm 0.02$ \\
Pleomorphic liposarcoma (PL) & 6 & $5.90 \pm 3.20$ \\
Leiomyosarcoma & 14 & $5.77 \pm 3.98$ \\
Desmoid tumors (DT) & 19 & $5.76 \pm 5.54$ \\
Myxoid liposarcoma (ML) & 7 & $4.70 \pm 2.33$ \\
Inflammatory myofibroblastic tumor (IMT) & 2 & $2.83 \pm 0.95$ \\
Well-differentiated liposarcoma (WDL) & 9 & $2.48 \pm 0.88$ \\
Round cell liposarcoma (RCL) & 1 & 47.3 \\
Adult fibrosarcoma (AF) & 1 & 25.94 \\
Malignant hemangiopericytoma (MH) & 1 & 11.21 \\
Follicular dendritic cell sarcoma (FDCS) & 1 & 7.3 \\
Primitive neuroectodermal tumor (PNET) & 1 & 7 \\
Malignant peripheral nerve sheath tumor & 1 & 6.4 \\
(MPNST) & 1 & 5.4 \\
Dermatofibrosarcoma protuberans (DFSP) & 1 & 5.19 \\
Rhabdomyosarcoma & 1 & 2.7 \\
Chondrosarcoma & &
\end{tabular}

\section{Benign tumors}

Pheochromocytoma and paraganglioma (PPGL) $13 \quad 7.87 \pm 5.26$

Schwannoma

Ganglioneuroma

Leiomyoma

Neurofibroma

Mature cystic teratoma (MST)

Hamartoma

$6 \quad 4.56 \pm 1.59$

$3 \begin{array}{ll}6 & 4.13 \pm 1.53\end{array}$

$2 \quad 1.05 \pm 1.48$

$1 \quad 5.5$

Hemangioma

2.6

$1 \quad 2.5$

\section{Psuedotumor}

Chronic abscess (CA)

Infection of actinomyces (IA)

Sarcoidosis

$1 \quad 2.2$

Hematoma

$2 \quad 14.4 \pm 8.77$

$1 \quad 14.16$

Reproductive tumors

High-grade serous ovarian carcinoma (HGSOC) $2 \quad 10.1 \pm 2.68$

Yolk sac tumor (YST)

Ovarian granulosa cell tumor (OGCT)

Dysgerminoma

Seminoma

9.75

$1 \quad 1.9$

\section{Others}

Peritoneal mesothelioma (PM)

$2 \quad 6.8 \pm 0.42$

Lymphoma

Malignant melanoma (MM)

Solid pseudopapillary tumor (SPT)

Perivascular epithelioid cell tumor (PEComa)

Lymph node metastasis (LM)

Carcinosarcoma

11.1

$1 \quad 10$

$1 \quad 4.1$

Sarcomatoid carcinoma (SCA)

$3 \quad 16.06 \pm$

0.92

$4 \quad 12.75 \pm$

8.56

$2 \quad 12.05 \pm$

0.35

$7.58 \pm 4.93$

$3 \quad 5.97 \pm 4.78$

$2 \quad 3.10 \pm 1.98$

13.1

$1 \quad 29.83$

myxoid liposarcoma, and well-differentiated liposarcoma often show lower SUVmax $[12,13]$. STS in group 2 are all assessed as FNCLCC grade 1 sarcoma, except for 1 myxoid liposarcoma patient (G2) and 1 desmoid tumor patient (G2). The SUVmax of the special myxoid liposarcoma and 
desmoid tumor are 4.3 and 6.47, respectively. We also have a special pleomorphic liposarcoma case assessed as grade 1, and its SUVmax is 2.5. We do not get enough proof to conclude dermatofibrosarcoma protuberans (DFSPs) and inflammatory myofibroblastic tumor (IMT) as the members of group 2, because we only have a bit G1 cases. In Aisheng Dong's study, the SUVmax of IMT was $10.9 \pm 5.5$, with a high variability of SUVmax among tumors ranging from 3.3 to 20.8 [14]. DFSP can also present high SUVmax [15]. Most reports about hematoma and lymph node metastasis focus on detection of lesions but not on differentiation with other diseases, so they did not list data of SUVmax $[16,17]$. SUVmax of hematoma and lymph node metastasis has been reported as 3.4 and 6.3 , but we still need more evidence $[18,19]$. We just temporarily regard them as group 2 members. On the other side, the range of PPGL SUVmax is from 2.5 to 62.3 [20]. Combined with our data, we list it as the only benign tumor in group 1 .

In the future, we think that the members of different groups may vary with the accumulation of cases. If we can establish such a mature system, it could be very helpful for the clinical use of 18F-FDG-PET/CT in retroperitoneal and intra-abdominal sarcomas. There will be 2 possibilities of this system. One is 2 different groups with certain diseases. The other is G1 sarcoma in one group, and rest sarcomas in another one. For now, we prefer the combination of these 2 possibilities, as certain disease is more likely to be of certain grade. If we can make the system mature, this differentiation must be very helpful for preoperative diagnosis combined with other examinations. For example, with exclusion of fatcontaining lesions using MRI, we should be very careful to perform compartment resection for group 2 diseases without liposarcoma.

For diagnostic aspect, SUVmax correlates with the Ki-67 index, mitotic count, and histological grade without considering different pathological types, which is the same as extremity STS [21]. This result suggests that 18F-FDGPET/CT may be helpful for preoperative biopsy and pathology dissection. For retroperitoneal sarcomas, it is reliable for core biopsy to determine the presence of a sarcoma, but it is difficult to correctly identify sarcoma subtype and grade $[2,22]$. Reason for this difficulty is the heterogeneity of sarcoma, which can be solved by multiple site sampling after resection. However, even sequential biopsies before resection cannot offer precise diagnosis for STS. The relationship of anatomic pathology and nuclear medicine mentioned in Manuel Scimeca's study has drawn our attention [23]. With the guidance of "SUVmax location," it may be helpful for core biopsy and pathology dissection to find the most representative part of a tumor. It is also possible to build a map of histological grade and different cell types. If the hypothesis is proved, it will reduce the number of biopsy and increase the accuracy of diagnosis and grade. The chaotic circumstance for STS diagnosis means that STS diagnosis and grade may vary with different biopsies, different samplings, or different pathologists. Even pathology of primary tumor and recurrent tumor in one patient could be different. Some relations may exist between different STS, like one STS changes into another one after several recurrences. However, we must know that any further studies or hypothesis must be established on accurate diagnosis and histological grade. With development of imaging fusion, we are convinced that the fusion of $18 \mathrm{~F}$ FDG-PET/CT and ultrasound will greatly enhance the accuracy of core biopsy and pathology dissection. This is also the aim for our further study.

For therapeutic aspect, SUVmax correlates with vascular resection but not with combined organ resection. This is because of our aggressive operative decisions. As there is high risk of thrombosis or bleeding, vascular resection is relatively passive. However, we will perform compartment resection even though some organs are "not really infiltrated" by tumors. At the same time, SUVmax does not correlate with pathological invasion of adjacent organs $(P=0.085)$, but the relationship is more significant than organ resection $(P=0.453)$. To some extent, we think SUVmax may be helpful to predict tumor infiltration and operative risks.

In prognostic aspect, SUVmax does correlate with STS prognostic factors including histological grade and recurrent tumors. However, we do not find the relationship among SUVmax, OS, DFS, death, and postoperative recurrence. This is because our follow-up is relatively short, and the median survival of STS is 103 months for R0 resection [24]. Our median follow-up is 10 months overall, with a range of 1 through 54 months. As SUVmax correlates with STS prognostic factors, we are convinced that we can get a positive result with enough follow-up in the future. For instance, G3 and recurrent sarcomas have higher SUVmax than G1 and primary sarcomas. G3 and recurrent sarcomas always leads to bad prognosis.

\section{Conclusion}

From our observation of retroperitoneal/intra-abdominal tumors, we draw the conclusion that 18F-FDG-PET/CT cannot simply distinguish malignant and benign tumors. We find that the SUVmax of malignant tumors, inflammatory pseudotumor, and PPGL group is higher than the SUVmax of benign tumors, lymph node metastasis, hematoma, and low malignant STS group. Guidance of "SUVmax location" may be helpful for biopsy and pathology dissection.

\section{Data Availability}

The data used to support the findings of this study are available from the corresponding author upon request.

\section{Conflicts of Interest}

The authors declare that they have no conflicts of interest.

\section{Acknowledgments}

This work was supported by the Beijing Municipal Administration of Hospital's Ascent Plan (Approval no. DFL20181104), Beijing Municipal Administration of Hospital's Clinical Medicine Development of Special Funding Support (Approval no. XMLX201708), Capital Health 
Research and Development of Special Funds (Approval no. 2016-2-2151), and National Natural Science Fund (Approval no. 31770836). The authors thank Si-meng Zou's support in drafting the manuscript.

\section{References}

[1] A. Kirane and A. M. Crago, "The importance of surgical margins in retroperitoneal sarcoma," Journal of Surgical Oncology, vol. 113, no. 3, pp. 270-276, 2016.

[2] S. Y. Hwang, S. Warrier, S. Thompson, T. Davidson, J. L. Yang, and P. Crowe, "Safety and accuracy of core biopsy in retroperitoneal sarcomas," Asia-Pacific Journal of Clinical Oncology, vol. 12, no. 1, pp. e174-e178, 2016.

[3] J. P. A. Ioannidis and J. Lau, "18F-FDG PET for the diagnosis and grading of soft-tissue sarcoma: a meta-analysis," Journal of Nuclear Medicine Official Publication Society of Nuclear Medicine, vol. 44, pp. 717-724, 2003.

[4] Z. Németh, K. Boér, and K. Borbély, "Advantages of (18)F FDG-PET/CT over conventional staging for sarcoma patients," Pathology \& Oncology Research, vol. 4, pp. 1-6, 2017.

[5] T. C. Kwee, S. Basu, B. Saboury, V. Ambrosini, D. A. Torigian, and A. Alavi, "A new dimension of FDG-PET interpretation: assessment of tumor biology," European Journal of Nuclear Medicine and Molecular Imaging, vol. 38, no. 6, pp. 11581170, 2011.

[6] Y. Yamamoto, Y. Ono, F. Aga, N. Kawai, N. Kudomi, and Y. Nishiyama, "Correlation of 18F-FLT uptake with tumor grade and Ki-67 immunohistochemistry in patients with newly diagnosed and recurrent gliomas," Journal of Nuclear Medicine, vol. 53, no. 12, pp. 1911-1915, 2012.

[7] D. Callegaro, R. Miceli, and R. Gladdy, "Prognostic models for RPS patients-attempting to predict patient outcomes," Journal of Surgical Oncology, vol. 117, no. 1, pp. 69-78, 2017.

[8] Y. Zhou, W. Hu, P. Chen et al., "Ki67 is a biological marker of malignant risk of gastrointestinal stromal tumors: a systematic review and meta-analysis," Medicine, vol. 96, no. 34, article e7911, 2017.

[9] M. C. Tan, M. F. Brennan, D. Kuk et al., "Histology-based classification predicts pattern of recurrence and improves risk stratification in primary retroperitoneal sarcoma," Annals of Surgery, vol. 263, no. 3, pp. 593-600, 2016.

[10] M. M. Von, R. L. Randall, R. S. Benjamin et al., "Soft tissue sarcoma, version 2.2016, NCCN clinical practice guidelines in oncology," Journal of the National Comprehensive Cancer Network, vol. 14, pp. 758-786, 2016.

[11] M. H. M. Schwarzbach, A. Dimitrakopoulou-Strauss, F. Willeke et al., "Clinical value of [18-F] fluorodeoxyglucose positron emission tomography imaging in soft tissue sarcomas," Annals of Surgery, vol. 231, no. 3, pp. 380-386, 2000.

[12] W. Brenner, J. F. Eary, W. Hwang, C. Vernon, and E. U. Conrad, "Risk assessment in liposarcoma patients based on FDG PET imaging," European Journal of Nuclear Medicine and Molecular Imaging, vol. 33, no. 11, pp. 1290-1295, 2006.

[13] H. Xu, H. J. Koo, S. Lim et al., "Desmoid-type fibromatosis of the thorax: CT, MRI, and FDG PET characteristics in a large series from a tertiary referral center," Medicine, vol. 94, no. 38, article e1547, 2015.

[14] A. Dong, Y. Wang, H. Dong et al., "Inflammatory myofibroblastic tumor: FDG PET/CT findings with pathologic correlation," Clinical Nuclear Medicine, vol. 39, no. 2, pp. 113-121, 2014.

[15] S. Basu and F. Goliwale, "18F-FDG PET/CT prediction of an aggressive clinical course for dermatofibrosarcoma protuberans,"
Journal of Nuclear Medicine Technology, vol. 44, no. 2, pp. 88-89, 2015.

[16] M. Atri, Z. Zhang, F. Dehdashti et al., "Utility of PET-CT to evaluate retroperitoneal lymph node metastasis in advanced cervical cancer: results of ACRIN6671/GOG0233 trial," Gynecologic Oncology, vol. 142, no. 3, pp. 413-419, 2016.

[17] A. Toriihara, E. Yamaga, M. Nakadate, J. Oyama, and U. Tateishi, "Detection of unexpected emergency diseases using FDG-PET/CT in oncology patients," Japanese Journal of Radiology, vol. 35, no. 9, pp. 539-545, 2017.

[18] G. Schaiberger, D. Pucar, V. Patel, B. Bateson, H. Williams, and W. Bates, "Para-atrial non-acute mediastinal hematoma after left atrial maze procedure mimicking tumor in a patient with treated melanoma," Journal of Nuclear Cardiology, vol. 4, pp. 1-3, 2017.

[19] Y. Tsunoda, M. Ito, H. Fujii, H. Kuwano, and N. Saito, "Preoperative diagnosis of lymph node metastases of colorectal cancer by FDG-PET/CT," Japanese Journal of Clinical Oncology, vol. 38, no. 5, pp. 347-353, 2008.

[20] T. H. Tan, Z. Hussein, F. F. Saad, and I. L. Shuaib, "Diagnostic performance of (68)Ga-DOTATATE PET/CT, (18)F-FDG PET/CT and (131)I-MIBG scintigraphy in mapping metastatic pheochromocytoma and paraganglioma," Nuclear Medicine and Molecular Imaging, vol. 49, no. 2, pp. 143-151, 2015.

[21] L. P. Adler, H. F. Blair, J. T. Makley et al., "Noninvasive grading of musculoskeletal tumors using PET," Journal of Nuclear Medicine Official Publication Society of Nuclear Medicine, vol. 32, no. 8, pp. 1508-1512, 1991.

[22] D. Berger-Richardson and C. J. Swallow, "Needle tract seeding after percutaneous biopsy of sarcoma: risk/benefit considerations," Cancer, vol. 123, no. 4, pp. 560-567, 2017.

[23] M. Scimeca, N. Urbano, R. Bonfiglio, O. Schillaci, and E. Bonanno, "Management of oncological patients in the digital era: anatomic pathology and nuclear medicine teamwork," Future Oncology, vol. 14, no. 11, pp. 1013-1015, 2018.

[24] J. J. Lewis, D. Leung, J. M. Woodruff, and M. F. Brennan, "Retroperitoneal soft-tissue sarcoma: analysis of 500 patients treated and followed at a single institution," Annals of Surgery, vol. 228, no. 3, pp. 355-365, 1998. 


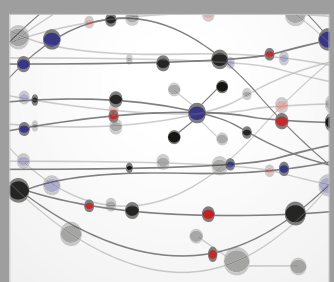

The Scientific World Journal
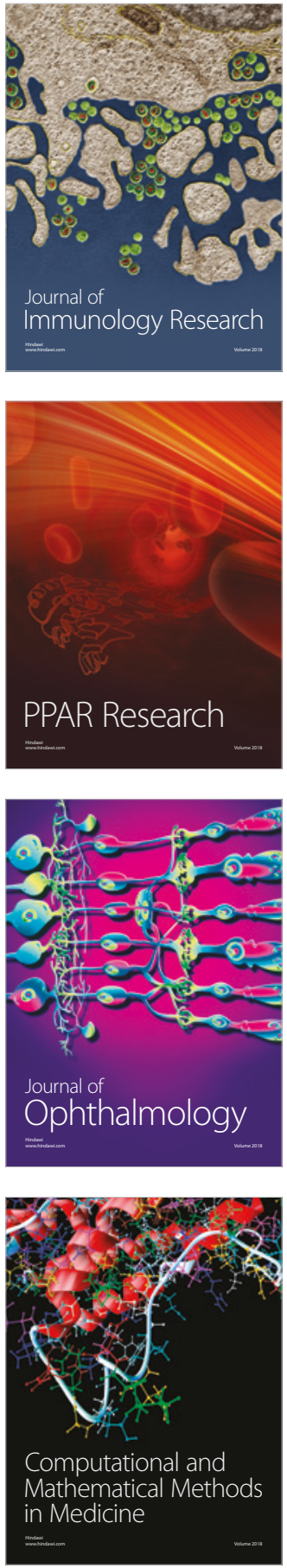

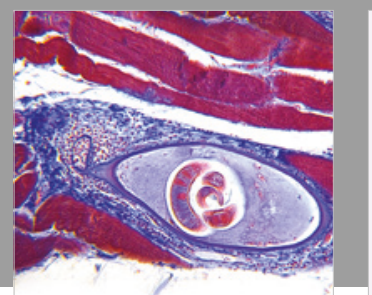

Gastroenterology Research and Practice

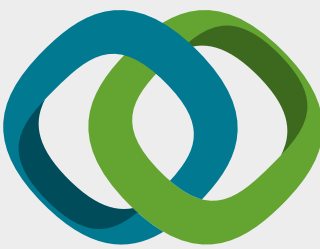

\section{Hindawi}

Submit your manuscripts at

www.hindawi.com
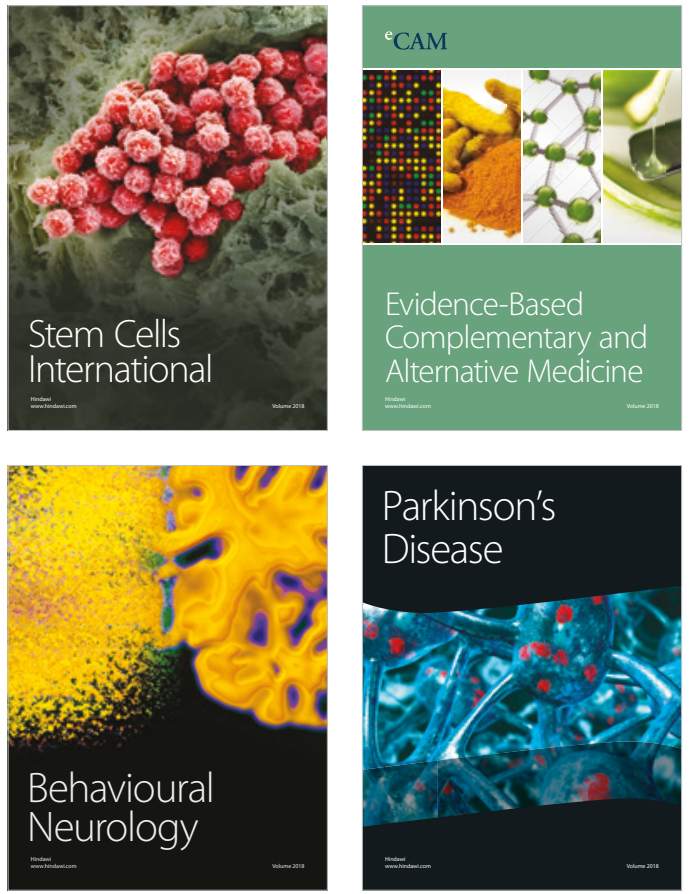

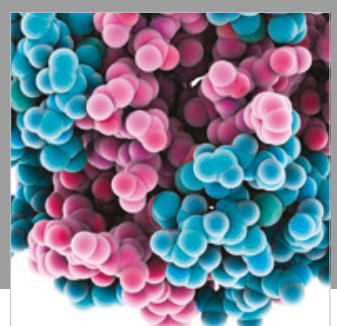

ournal of

Diabetes Research

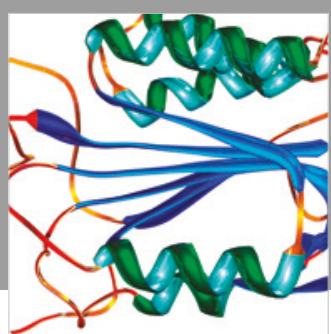

Disease Markers
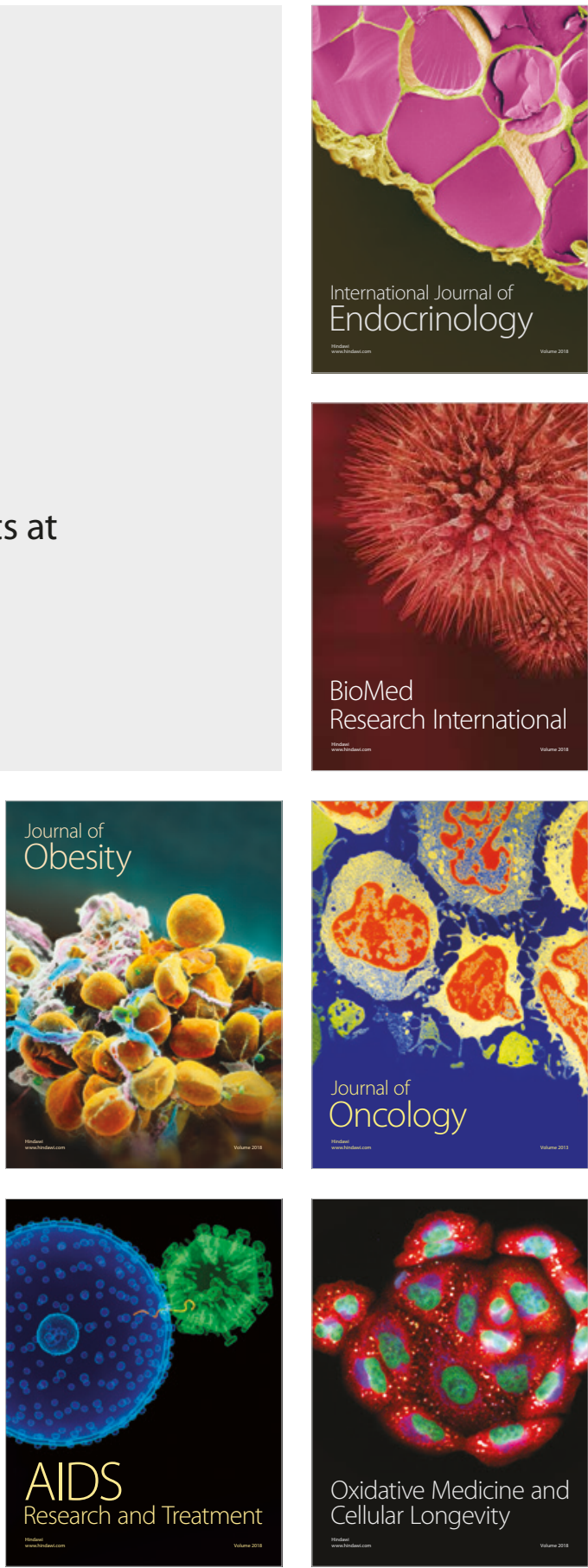